\title{
Autonomic Neuropathy in HIV: A Case Report and Review of Potential Symptoms in an Advanced-Stage, HIV Cohort
}

\author{
Jessica Robinson-Papp, Kathryn J. Elliott, Maria Pizzirusso, Susan Morgello
}

Department of Neurology, Mount Sinai School of Medicine, New York, USA.

Email: jessica.robinson-papp@mssm.edu

Received July $19^{\text {th }}$, 2012; revised August $19^{\text {th }}$, 2012; accepted August $27^{\text {th }}, 2012$

\begin{abstract}
A woman with a seventeen-year history of HIV infection on effective antiretroviral therapy presented with signs and symptoms of distal symmetric polyneuropathy (DSP). She developed significant side effects (dizziness, nausea, edema) with medications for pain management. Chart review revealed a history of similar intolerance to multiple antiretrovirals, which was not explained by known cardiac or gastrointestinal disease or psychological factors. Specialized testing revealed the presence of an autonomic neuropathy, which provided an explanation for her medication intolerance. The patient was educated on the symptoms of autonomic neuropathy and its relationship to DSP and subsequent symptommatic medications were initiated at the lowest possible doses. Although symptom management remained challenging, the patient exhibited lower frustration and greater acceptance of medication trials. Review of 168 advanced stage HIV infected individuals demonstrated that $81 \%$ experienced at least one, and $33 \%$ three or more, symptoms potentially attributable to autonomic neuropathy. Potentially autonomic symptoms were significantly associated with the presence of symptomatic DSP. Autonomic neuropathy is difficult to diagnose without specialized testing, as its symptoms are non-specific and overlap with a large number of somatic disorders. The high prevalence of autonomic-type symptoms in chronic HIV, and their association with peripheral neuropathy, may warrant further investigation of the potential for autonomic dysfunction in individuals with HIV-related symptomatic DSP.
\end{abstract}

Keywords: HIV; Neuropathy; Autonomic

\section{Introduction}

HIV-associated distal symmetric polyneuropathy (HIVDSP) is a common complication of HIV, and its symptoms include pain, paresthesias and numbness, usually beginning symmetrically in the feet [1,2]. Recent publications have documented the continued high prevalence of HIV-DSP and its impact on quality of life. It is estimated that between $30 \%-60 \%$ of HIV-infected individuals have signs of HIV-DSP and that neuropathic pain is associated with unemployment, poorer quality of life and reduced ability to perform activities of daily living $[1,3]$. Dysfunction of small caliber nociceptive nerve fibers (c-fibers) is thought to underlie the pain of HIVDSP, and loss of small fibers has been demonstrated in skin biopsies [4]. Autonomic nerves are also comprised of small fibers, and are responsible for control of crucial homeostatic mechanisms such as regulation of heart rate and blood pressure, gastrointestinal motility, urination and thermoregulation. Autonomic neuropathy is wellknown to accompany DSP in other diseases such as diabetes. However, symptoms of autonomic neuropathy such as orthostatic dizziness or fainting, nausea or vomiting especially with meals, diarrhea and/or constipation, urinary incontinence, sexual dysfunction, and changes in sweating, are not typically thought of as part of the HIVDSP symptom complex. These symptoms seldom elicit a diagnostic work up for autonomic dysfunction, as they are thought to more commonly be aspects of systemic illnesses or medication intolerance occurring in the context of HIV. Herein, we present a patient with HIV-DSP, who was subsequently found to have an associated, unrecognized autonomic neuropathy that may have accounted for her systemic symptoms and intolerance to multiple medications. We review potentially autonomic symptoms and their relationship to HIV-DSP in 168 patients followed by the Manhattan HIV Brain Bank, a longitudinal study of advanced-stage, chronically HIV-infected individuals with high prevalence of antiretroviral therapy and HIV-DSP.

\section{Case Report}

The patient was a 46 year-old HIV-infected woman who 
had acquired HIV 17 years previously via heterosexual transmission. She was adherent to her antiretroviral regimen of abacavir-lamivudine and atazanavir, which had been unchanged for about 18 months. She had been on multiple previous antiretroviral regimens (over 15 different agents), which had to be discontinued due to side effects, the most severe of which was intractable nausea and vomiting. No specific diagnostic evaluation had been performed because the symptoms were presumed to be medication related. Current CD4+ count was 257 cells $/ \mathrm{mm}^{3}$ and HIV-1 viral load was undetectable, CD4+ nadir was 164 cells $/ \mathrm{mm}^{3}$ at the time of initial HIV diagnosis. She had mild hypertension, but no other active medical co-morbidities, and did not use alcohol or other substances. Over the course of one year the patient developed worsening pain in her feet, for which she was referred to the neurology clinic. Neurologic examination revealed hyperalgesia and allodynia in the soles of both feet and decreased pinprick sensation up to the distal calf bilaterally, and was otherwise normal. Nerve conduction studies and electromyography were normal. Laboratory testing revealed no evidence of diabetes mellitus, hypothyroidism, vitamin B12 deficiency, or monoclonal gammopathy. A presumptive diagnosis of small fiber neuropathy was made and the patient was started on a low dose of gabapentin (100 mg PO TID) because of the history of difficulty tolerating medications. She developed lower extremity edema and dizziness. Gabapentin was discontinued and duloxetine $20 \mathrm{mg}$ daily was started. The patient developed intolerable nausea and dizziness. Duloxetine was discontinued and nortiptyline $10 \mathrm{mg}$ QHS was started, with hydrocodone-acetaminophen 5 - 500 mg once a day in the afternoon. She developed marked constipation, but this was resolved with oral senna and docusate. Due to the difficulty in treating her, additional testing was pursued to confirm the diagnosis of small fiber neuropathy. An autonomic reflex screen was chosen over skin biopsy because it is a non-invasive measure of small fiber function. Testing included the quantitative sudomotor axon reflex test (QSART), heart rate response to deep breathing (HRDB), Valsalva maneuver (VM) and tilt table testing. [5] All measures were abnormal indicating moderately severe autonomic neuropathy involving sudomotor, sympathetic and parasympathetic systems. During the tilt table she exhibited orthostatic hypotension, without adequate compensatory tachycardia and was nearly syncopal (see Figure 1). In light of these results, further inquiry was made for possible autonomic symptoms that could have been overlooked, and her records over the past 3 years were reviewed. Chart review confirmed intolerance to multiple medications with symptoms of dizziness or nausea and vomiting, as well as uncontrollable diarrhea in response to darunavir and tenofovir/emtricitabine two years prior to her neurologic presentation. Earlier that year, she had been diagnosed with "dyspepsia" and started on esomeprazole. Current symptoms elicited by interview and administration of the Autonomic Symptoms Profile [6], a validated questionnaire, included: lightheadedness, dry mouth, changes in sweating, nausea and bloating after a small meal, urinary urgency with stress incontinence, and light sensitivity. Following the diagnosis of autonomic neuropathy, the patient was educated on its signs and symptoms and its relationship to DSP. Subsequent symptomatic medications were initiated at the lowest possible doses with close follow-up for worsening of orthostatic hypotension or nausea. Although her neuropathic pain symptoms remained difficult to treat, understanding the underlying autonomic neuropathy provided the patient some reassurance and enhanced her acceptance of medication trials. If a tolerable neuropathic pain treatment cannot be found, the next step in management will be the addition of ancillary treatments specifically directed at side effects such as lower extremity compression stockings to combat venous pooling and edema, reduction of antihypertensives to reduce orthostatic hypotension, and addition of prokinetic agents to enhance gastric motility and reduce nausea and vomiting.

\section{Potentially Autonomic Symptoms: Prevalence in an Advanced Stage, HIV Cohort}

The Manhattan HIV Brain Bank (MHBB) is an ongoing,
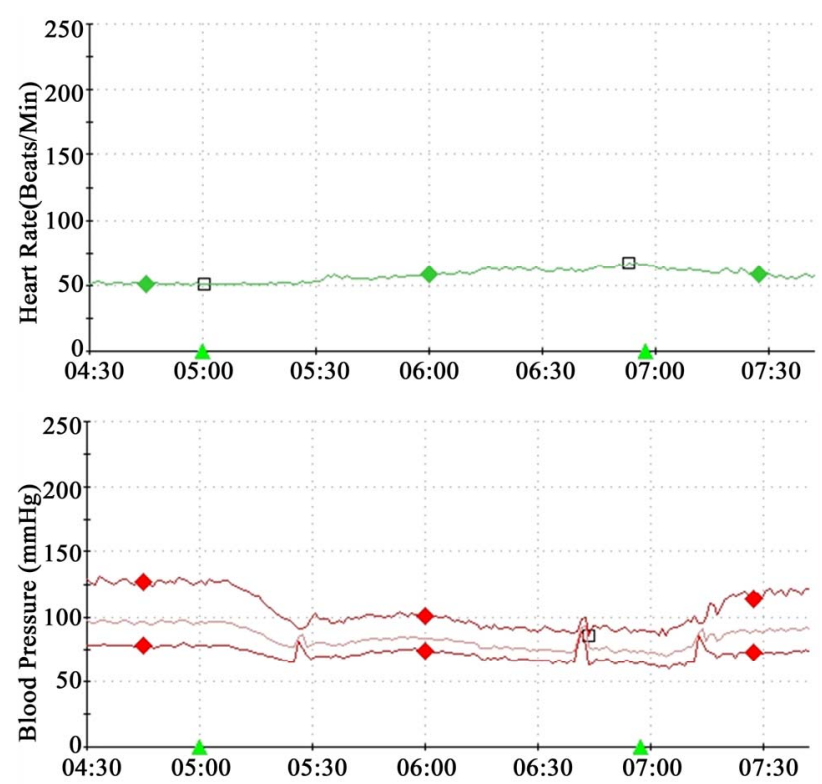

Figure 1. Data from a tilt table test reveals orthostatic hypotension, without adequate compensatory tachycardia, in a patient presenting with painful HIV-associated distal symmetric polyneuropathy. The patient was upright in the time period between the triangles (minutes 5 - 7). This was one of four autonomic reflex tests performed. All were abnormal, confirming the presence of autonomic neuropathy. 
prospective nervous system study of participants with long-standing HIV, as described previously. [7] All participants seen between 1/1/2009 and 11/15/2011 were reviewed for signs of HIV-DSP and potential symptoms of autonomic dysfunction. There were 168 complete, distinct records. Each visit consisted of a structured medical history including review of antiretrovirals and co-morbid illnesses relevant to DSP (e.g. diabetes mellitus); measurement of CD4+ count and HIV-1 viral load; and a comprehensive neurological examination performed by an attending neurologist. A diagnosis of symptomatic DSP was assigned if a participant reported paresthesias, dysesthesias or numbness in a distal distribution and displayed at least two of the following three signs: diminished sense of vibration at both great toes, bilateral distal decreased sensation to pin prick in both lower extremities, and ankle reflexes absent or diminished as compared to the knees. An extensive review of systems was performed as part of the general neuromedical assessment and contained the following potentially autonomic symptoms: syncope or fainting, urinary incontinence, fecal incontinence, diarrhea, changes in appetite, changes in sleep and pupillary abnormalities.

The great majority (94\%) of participants were receiving antiretroviral therapy including: nucleoside reverse transcriptase inhibitors (87\%), non-nucleoside reverse transcriptase inhibitors (31\%), protease inhibitors (61\%), entry inhibitors (4\%) and integrase inhibitors (21\%). Sixty-seven participants (40\%) had HIV-DSP (see Table 1). Many other neurologic conditions were also observed. Seventy-eight percent of participants had at least mild cognitive impairment detected on neuropsychological testing. Fifty-three percent had a neurologic diagnosis other than HIV-DSP or neurocognitive impairment. These included cerebrovascular disease, cranial nerve abnormalities, Parkinsonism, tremor, visual impairment, herpes zoster, and radiculopathy. These disorders were typically medically managed according to standard of care, although specific data about surgical interventions was not collected in a standardized fashion.

Potentially autonomic symptoms were common, with 136 (81\%) participants experiencing at least one symptom, 38 (23\%) experiencing two, and 55 (33\%) experiencing three or more. The presence of one or more of the above symptoms was associated with HIV-DSP in a Chi-square analysis $\left(\chi^{2}(1)=7.4, p=0.007\right)$. Among participants with one or more of these symptoms, $45 \%$ had HIV-DSP compared to $19 \%$ among the remaining participants. The presence of one or more of these symptoms was also associated with longer duration of HIV infection: 19 years (IQR 15.21) vs 16 years (IQR 12.19) (Wilcoxon rank-sum $\left(\mathrm{n}_{1}=136, \mathrm{n}_{2}=31\right)=1955, \mathrm{p}=$ 0.007); but not with: age, gender, ethnicity, current CD4+ count, a detectable HIV viral load, the use of an
Table 1. Characteristics of Manhattan HIV Brain Bank Cohort ${ }^{\mathrm{a}}$.

\begin{tabular}{|c|c|}
\hline $\mathrm{N}$ & 168 \\
\hline HIV-DSP & $40 \%$ \\
\hline Age, years & $52(47,57)$ \\
\hline \multicolumn{2}{|l|}{ Gender } \\
\hline Female & $55 \%$ \\
\hline Male & $45 \%$ \\
\hline \multicolumn{2}{|l|}{ Ethnicity } \\
\hline White & $18 \%$ \\
\hline African-American/Black & $50 \%$ \\
\hline Hispanic/Latino & $30 \%$ \\
\hline Other & $2 \%$ \\
\hline HIV duration, years & $19(14,21)$ \\
\hline Log (VL, copies/ml) & $1.7(1.7,3.0)$ \\
\hline CD4+ (cells $\left./ \mathrm{mm}^{3}\right)$ & $397(195,616)$ \\
\hline Prescribed ART & $93 \%$ \\
\hline \multicolumn{2}{|l|}{ Symptom prevalence } \\
\hline Syncope & $11 \%$ \\
\hline Urinary incontinence & $30 \%$ \\
\hline Fecal incontinence & $14 \%$ \\
\hline Diarrhea & $22 \%$ \\
\hline Changes in sleep & $57 \%$ \\
\hline Changes in appetite & $50 \%$ \\
\hline Pupil changes & $14 \%$ \\
\hline
\end{tabular}

${ }^{a}$ Values are median (interquartile range), unless indicated as a percentage. Abbreviations: HIV-DSP = HIV-associated distal symmetric polyneuropathy; $\mathrm{VL}$ = viral load; ART = antiretroviral treatment.

tiretrovirals (in general or by class), or a diagnosis of diabetes mellitus (all p-values $>0.1$ in Chi-square or the Wilcoxon rank sum test as appropriate). The association between the presence of one or more potentially autonomic symptoms and both duration of HIV infection (OR $=1.1$; 95\%CI: 1.0, 1.2; $\mathrm{p}=0.01)$ and HIV-DSP $(\mathrm{OR}=$ 3.7; 95\%CI: 1.3, 10.1; $\mathrm{p}=0.01$ ) was maintained in a multivariate logistic regression model adjusting for age, antiretroviral use and diabetes mellitus.

\section{Discussion}

This report describes a commonly encountered clinical scenario, distal neuropathic pain due to HIV-DSP. There is a high level of awareness in the HIV community about the prevalence of HIV-DSP and its signs and symptoms. However there is very little awareness that HIV-DSP may be accompanied by autonomic neuropathy, as exemplified by our case report. This patient had symptoms that were fairly typical of autonomic dysfunction, but remained undiagnosed, perhaps because her symptoms 
were relatively mild until exacerbated by medication. In her case, the development of painful HIV-DSP finally led to the diagnosis of her autonomic neuropathy.

It is unknown what proportion of patients with HIVDSP have autonomic neuropathy, although recent studies have documented an association between autonomic neuropathy and HIV [8-10] and studies from early in the HIV epidemic suggested that autonomic neuropathy was in fact quite common [11,12]. In our review of participants in the MHBB, potentially autonomic symptoms were found far more frequently in participants with symptomatic HIV-DSP than those without. Without formal autonomic testing it is impossible to say whether or not these symptoms were truly due to autonomic neuropathy. However the symptoms were not associated with current disease severity as measured by CD4+ count and HIV-1 viral load, suggesting that they were not readily explained by HIV alone. Similarly, the lack of association with antiretrovirals suggests that these symptoms cannot be explained by toxicity alone.

Raising awareness about the symptoms of autonomic neuropathy is complicated by the diverse and non-specific nature of autonomic symptoms, and the difficulty in proving that any given symptom is autonomic. The complexity of assessing autonomic symptoms is illustrated by the Autonomic Symptom Profile (ASP), the most extensively used measure of autonomic symptoms [6]. The ASP contains 169 items which query a wide variety of symptoms including: positional and situational dizziness and syncope; skin changes including color changes (due to vasomotor instability) and changes in sweat output; dry eyes and mouth; gastrointestinal symptoms such as nausea, vomiting or bloating (particularly after eating), diarrhea or constipation; urinary incontinence or hesitancy; sexual dysfunction; sensitivity to light or blurry vision (due to dysfunction of pupillary constriction); and sleep disturbance. Even patients with florid autonomic failure do not typically experience all of these symptoms and patients with milder disease may have only one or two, which may make appreciation of an underlying autonomic disorder particularly challenging.

In the case of our patient, autonomic neuropathy likely predated her HIV-DSP symptoms and could have accounted for the numerous side effects she experienced from antiretrovirals. This would have been important information for her care providers that might have altered their prescribing strategy and led to exposure to fewer antiretrovirals and greater attention to providing symptomatic relief. Further research is needed to determine the prevalence of autonomic neuropathy in HIV and its relationship to HIV-DSP.

\section{Acknowledgements}

The authors thank the staff and participants of the Man- hattan HIV Brain Bank. Dr. Robinson-Papp receives funding from NINDS (K23NS066789) and Dr. Morgello receives funding from NIMH and NINDS (U01MH083501, R24MH59724).

\section{REFERENCES}

[1] R. J. Ellis, D. Rosario, D. B. Clifford, et al., "Continued High Prevalence and Adverse Clinical Impact of Human Immunodeficiency Virus-Associated Sensory Neuropathy in the Era of Combination Antiretroviral Therapy: The CHARTER Study," Archives of Neurology, Vol. 67, No. 5, 2010, pp. 552-558. doi:10.1001/archneurol.2010.76

[2] J. Robinson-Papp, B. Gelman, I. Grant, E. Singer, G. Gensler and S. Morgello, "Substance Abuse Increases the Risk of Neuropathy in an HIV-Infected Cohort," Muscle Nerve, Vol. 45, No. 4, 2012, pp. 471-476. doi:10.1002/mus.23231

[3] S. R. Evans, R. J. Ellis, H. Chen, et al., "Peripheral Neuropathy in HIV: Prevalence and Risk Factors," AIDS, Vol. 25, No. 7, 2011, pp. 919-928. doi:10.1097/QAD.0b013e328345889d

[4] M. Polydefkis, C. T. Yiannoutsos, B. A. Cohen, et al., "Reduced Intraepidermal Nerve Fiber Density in HIVAssociated Sensory Neuropathy,” Neurology, Vol. 58, No. 1, 2002, pp. 115-119. doi:10.1212/WNL.58.1.115

[5] P. A. Low, "Composite Autonomic Scoring Scale for Laboratory Quantification of Generalized Autonomic Failure,” Mayo Clinic Proceedings, Vol. 68, No. 8, 1993, pp. 748-752.

[6] G. A. Suarez, T. L. Opfer-Gehrking, K. P. Offord, E. J. Atkinson, P. C. O’Brien and P. A. Low, "The Autonomic Symptom Profile: A New Instrument to Assess Autonomic Symptoms,” Neurology, Vol. 52, No. 3, 1999, pp. 523-528. doi:10.1212/WNL.52.3.523

[7] S. Morgello, L. Estanislao, D. Simpson, et al., "HIVAssociated Distal Sensory Polyneuropathy in the Era of Highly Active Antiretroviral Therapy: The Manhattan HIV Brain Bank,” Archives of Neurology, Vol. 61, No. 4, 2004, pp. 546-551. doi:10.1001/archneur.61.4.546

[8] C. Compostella, L. Compostella and R. D’Elia, “Cardiovascular Autonomic Neuropathy in HIV-Positive African Patients," Minerva Cardioangiologica, Vol. 56, No. 4, 2008, pp. 417-428.

[9] D. C. Chow, R. Wood, J. Choi, et al., "Cardiovagal Autonomic Function in HIV-Infected Patients with Unsuppressed HIV Viremia,” HIV Clinical Trials, Vol. 12, No. 3, 2011, pp. 141-150. doi:10.1310/hct1203-141

[10] G. Askgaard, U. S. Kristoffersen, J. Mehlsen, G. Kronborg, A. Kjaer and A. M. Lebech, "Decreased Heart Rate Variability in HIV Positive Patients Receiving Antiretroviral Therapy: Importance of Blood Glucose and Cholesterol,” PLoS One, Vol. 6, No. 5, 2011, Article ID: e20196. doi:10.1371/journal.pone.0020196

[11] R. Freeman, M. S. Roberts, L. S. Friedman and C. Broadbridge, "Autonomic Function and Human Immunodeficiency Virus Infection,” Neurology, Vol. 40, No. 4, 1990, pp. 575-580. doi:10.1212/WNL.40.4.575 
[12] S. Ruttimann, P. Hilti, G. A. Spinas and U. C. Dubach, "High Frequency of Human Immunodeficiency VirusAssociated Autonomic Neuropathy and More Severe Involvement in Advanced Stages of Human Immunodefi- ciency Virus Disease," Archives of Internal Medicine, Vol. 151, No. 12, 1991, pp. 2441-2443.

doi:10.1001/archinte.1991.00400120079013 